\title{
Intrinsic functional network organization in high-functioning adolescents with autism spectrum disorder
}

\author{
Elizabeth Redcay ${ }^{*}$, Joseph M. Moran ${ }^{2,3}$, Penelope L. Mavros ${ }^{4}$, Helen Tager-Flusberg ${ }^{5}$, \\ John D. E. Gabrieli ${ }^{6,7}$ and Susan Whitfield-Gabrieli ${ }^{6,7}$
}

${ }^{1}$ Department of Psychology, University of Maryland, College Park, MD, USA

${ }^{2}$ Center for Brain Science, Harvard University, Cambridge, MA, USA

${ }^{3}$ United States Army Natick Soldier Research Development and Engineering Center, Natick, MA, USA

${ }^{4}$ Simons Center for the Social Brain at Massachusetts Institute of Technology, Cambridge, MA, USA

${ }^{5}$ Department of Psychology, Boston University, Boston, MA, USA

${ }^{6}$ McGovern Institute for Brain Research at Massachusetts Institute of Technology, Cambridge, MA, USA

${ }^{7}$ Department of Brain and Cognitive Sciences, Massachusetts Institute of Technology, Cambridge, MA, USA

\section{Edited by:}

Ralph-Axel Müller, San Diego State

University, USA

\section{Reviewed by:}

Christian Sorg, Klinikum rechts der Isar Technische Universität München, Germany

Michal Assaf, Institute of Living, USA

\section{${ }^{*}$ Correspondence:}

Elizabeth Redcay, Department of Psychology, University of Maryland, 1147 Biology-Psychology Building, College Park, MD 20742, USA

e-mail: redcay@umd.edu
Converging theories and data suggest that atypical patterns of functional and structural connectivity are a hallmark neurobiological feature of autism. However, empirical studies of functional connectivity, or, the correlation of MRI signal between brain regions, have largely been conducted during task performance and/or focused on group differences within one network [e.g., the default mode network (DMN)]. This narrow focus on task-based connectivity and single network analyses precludes investigation of whole-brain intrinsic network organization in autism. To assess whole-brain network properties in adolescents with autism, we collected resting-state functional connectivity MRI (rs-fcMRI) data from neurotypical (NT) adolescents and adolescents with autism spectrum disorder (ASD). We used graph theory metrics on rs-fcMRI data with 34 regions of interest (i.e., nodes) that encompass four different functionally defined networks: cingulo-opercular, cerebellar, fronto-parietal, and DMN (Fair et al., 2009). Contrary to our hypotheses, network analyses revealed minimal differences between groups with one exception. Betweenness centrality, which indicates the degree to which a seed (or node) functions as a hub within and between networks, was greater for participants with autism for the right lateral parietal (RLatP) region of the DMN. Follow-up seed-based analyses demonstrated greater functional connectivity in ASD than NT groups between the RLatP seed and another region of the DMN, the anterior medial prefrontal cortex. Greater connectivity between these regions was related to lower ADOS (Autism Diagnostic Observation Schedule) scores (i.e., lower impairment) in autism. These findings do not support current theories of underconnectivity in autism, but, rather, underscore the need for future studies to systematically examine factors that can influence patterns of intrinsic connectivity such as autism severity, age, and head motion.

Keywords: autism, resting-state functional connectivity, default mode network, intrinsic network organization, graph theory, functional MRI

\section{INTRODUCTION}

Atypical patterns of functional and structural connectivity are proposed to be a hallmark neurobiological feature of autism (Belmonte et al., 2004; Just et al., 2004; Courchesne and Pierce, 2005; Cherkassky etal., 2006). Most theories and data point to a pattern of underconnectivity, particularly for long-distance connections such as interhemispheric or anterior-posterior intrahemispheric connections (Belmonte et al., 2004; Just et al., 2004; Anderson et al., 2011; Dinstein et al., 2011). Some also suggest an increase in local connections at the expense of long-distance connections (Courchesne and Pierce, 2005; Courchesne et al., 2007; Rippon et al., 2007). Recent findings, however, offer mixed support and suggest a more complex picture of connectivity differences in autism with evidence for both hypo- and hyper-connectivity for short- and long-distance connections, depending partly on the specific experimental and analytic methods used and age of the participants (e.g., Courchesne etal., 2007; Noonan etal., 2009; Khan et al., 2013; Lynch et al., 2013; review, Müller et al., 2011).

Structural connectivity findings, indexed by measures of white matter integrity from diffusion tensor imaging (DTI) (e.g., fractional anisotropy, or FA) or white matter volumes from structural MRI, reveal atypical connectivity patterns in autism but do not support general underconnectivity in autism. Rather, findings suggest developmentally increased white matter volume (Courchesne et al., 2001; Hazlett et al., 2006), particularly radiate white matter bundles supporting interhemispheric and cortico-cortical connections (Herbert et al., 2004) and increased FA in infants and young children with autism (e.g., Ben Bashat etal., 2007; Wolff et al., 2012), whereas later in development (e.g., adolescents and adults), FA is decreased (e.g., Barnea-Goraly et al., 2004; Lee et al., 2007; Nair et al., 2013). 
Studies of functional connectivity, or the correlation in signal between brain regions, largely have supported the underconnectivity theory when functional connectivity has been assessed in the context of a task (review, Müller et al., 2011). This pattern of reduced long-distance connectivity (e.g., between regions of different hemispheres or lobes) is seen across domains of function including tasks involving language processing (e.g., Just et al., 2004; Kana et al., 2006), executive function (e.g., Just et al., 2007), and social processing (e.g., Mason et al., 2008; Kana et al., 2012; , but see Murphy et al., 2012), but notably these tasks also resulted in reduced activation in the autism spectrum disorder (ASD) group as compared to the neurotypical (NT) group. Thus, while informative, task-based functional connectivity analyses may reflect differences in performance during a task and may not reflect differences in intrinsic functional organization of the brain.

Task-independent studies of the "resting" brain provide a window with which to examine intrinsic functional network organization. As first noted by Biswal et al. (1995), even in the absence of a specific task, fluctuations in brain signal are temporally correlated within regions that are part of the same functional network. These large-scale functional networks can be identified using data-driven ICA (independent component analysis) analyses (e.g., Damoiseaux et al., 2006) or seed-based analyses (e.g., Fox et al., 2005) and are thought to reflect regions that have a history of co-activation. Indeed, differences in the organization or connection strength within these regions are related to developmental changes (e.g., Fair et al., 2009), training (Lewis et al., 2009), and individual differences, for example in memory (Wang et al., 2010), math abilities (Emerson and Cantlon, 2012), and face processing (Zhu et al., 2011), suggesting intrinsic network connectivity is behaviorally relevant.

There has been considerable divergence across studies in regards to the status of resting-brain functional connectivity in ASD. Like task-based studies, many studies of the resting brain in ASD (or those in which the task is used as a regressor of no interest) have revealed reduced functional connectivity in ASD, particularly for long-range connections (Cherkassky et al., 2006; Kennedy and Courchesne, 2008; Ebisch et al., 2011; Tsiaras et al., 2011; Murdaugh et al., 2012; Rudie et al., 2012; Washington et al., 2013). However, unlike task-based studies, a number of studies report findings that are inconsistent with a general theory of underconnectivity (e.g., Monk et al., 2009; Müller et al., 2011; Tyszka et al., 2013), and in some cases hyper-connectivity in ASD groups has been reported (Mizuno et al., 2006; Turner et al., 2006; Noonan et al., 2009; Di Martino et al., 2011; Shih et al., 2011; Lynch et al., 2013).

In sum, extant data suggest a general underconnectivity theory in autism is likely not the full story. Possibly, the age of the participant, the context in which connectivity is assessed (e.g., resting vs. task), and the specific networks examined may result in different findings between groups. Further, recent studies suggest that head motion may lead to systematic, spurious correlations which could mimic some of the same patterns of connectivity differences reported between autism and NT groups (Power et al., 2011). An incomplete picture of how each of these factors contributes to functional connectivity in autism still remains. One additional contributing factor is that most previous studies only focused on the strength of correlations within a single network rather than examining network organization with graph theoretical metrics. Recent advances in graph theory (or complex network) analyses for resting-state functional connectivity MRI (rs-fcMRI) data allow for characterization of whole-brain intrinsic network organization (e.g., review, Rubinov and Sporns, 2010; Bullmore and Bassett, 2011). Specifically, rather than focusing on the strength of region-region correlations, graph theory methods can examine the topological properties of each region within the context of all other regions of interest. For example, graph theory metrics can include measures of the integration (global efficiency, average path length), segregation (local efficiency, clustering coefficient), and centrality (betweenness centrality) of networks. Thus, these metrics can provide a more robust test of the theory of reduced long-distance and increased local connectivity by testing differences in measures of whole-brain network integration and segregation.

In the current study, we assessed whole-brain network properties in a group of adolescents with and without autism by using graph theory and seed-based analyses on rs-fcMRI data with functionally defined regions of interest. The functional regions of interest included 34 regions identified from previous metaanalyses (Dosenbach et al., 2006; Fair et al., 2009) that encompass four different functionally defined networks: cingulo-opercular (CO), cerebellar (C), fronto-parietal (FP), and default mode (DMN; Fair et al., 2009). These networks were chosen because previous research with these same networks has demonstrated a developmental pattern of progressive increases in long-distance connectivity between nodes of the same network and concurrent decreases in connectivity between anatomically proximal nodes of distinct networks (Fair et al., 2008, 2009). Furthermore, functions associated with these networks have all been implicated in autism (e.g., reviews, Di Martino et al., 2009; Minshew and Keller, 2010). Thus, examining these networks allows for a more rigorous test of the hypothesis of reduced long-distance and increased local connectivity in autism, across multiple networks that support varied functions.

\section{MATERIALS AND METHODS PARTICIPANTS}

All participants gave written, informed consent and parental consent was obtained for participants under 18 years of age as approved by the Committee on the Use of Humans as Experimental Subjects (COUHES) at the Massachusetts Institute of Technology. Participants were compensated monetarily for their time. Participants were part of a multi-site study involving three visits for TD adolescents and four for the ASD group but only the resting-state functional MRI data are presented in the current study. Participant IQ was measured using the Kaufman Brief Intelligence Test (KBIT-2).

\section{AUTISM SPECTRUM DISORDER PARTICIPANTS}

We collected resting-state functional MRI data from 22 male adolescents and young adults (14-20 years; mean $17.3 \pm 2.2$ years; all male) with a clinical diagnosis of ASD or Asperger's disorder. Diagnosis was confirmed using a combination of the 
Table 1 | Demographic and head motion information for NT and ASD groups and those ASD participants excluded due to excessive head motion.

\begin{tabular}{llllll}
\hline & NT $(\boldsymbol{N}=\mathbf{1 4})$ & ASD $(\boldsymbol{N}=\mathbf{1 4})$ & $\begin{array}{l}\text { ASD-excluded } \\
(\boldsymbol{N}=\mathbf{7})\end{array}$ & $\begin{array}{l}\text { NT vs. ASD } \\
(\boldsymbol{p} \text {-value })\end{array}$ & $\begin{array}{l}\text { ASD vs. ASD-excluded } \\
(\boldsymbol{p} \text {-value })\end{array}$ \\
\hline Age & $17.7(1.8)$ & $17.8(1.9)$ & $15.8(2.5)$ & 0.81 & 0.05 \\
Full Scale IQ & $119(9.6)$ & $116.9(13.7)$ & $98.3(24.4)$ & 0.59 & 0.04 \\
Verbal IO & $118(13.1)$ & $116.3(15.1)$ & $97(24.9)$ & 0.75 & 0.04 \\
Non-verbal IQ & $115(10.3)$ & $112.5(13.1)$ & $99.7(27.3)$ & 0.57 & 0.17 \\
Motion outliers & $2.2(3.8)$ & $1.8(2.8)$ & $45.9(17.3)$ & 0.73 & $<0.0001^{\circ}$ \\
ADOS Combined & N/A & $9.5(1.3)$ & $16.2(2.8)$ & N/A & 0.02 \\
ADOS Comm. & N/A & $3(2)$ & $4.2(2.3)$ & N/A & 0.28 \\
ADOS Social & N/A & $6.5(2.8)$ & $12(5.1)$ & N/A & 0.007
\end{tabular}

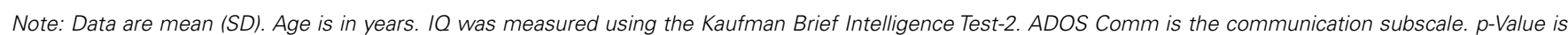
based on a t-test comparing groups. ${ }^{\circ}$ This difference is circular because these groups were created based on differences in motion outliers.

Autism Diagnostic Observation Schedule (ADOS) Module 3 or 4 (administered to the participant; Lord et al., 2000) and the Social Communication Questionnaire (SCQ; completed by the parent of the participant; Corsello et al., 2007). The SCQ is a questionnaire designed to screen for autism and all included ASD participants received an SCQ score greater than the suggested cut-off for ASD of 15 (mean 21.6; 16-28). All participants reached criteria for Autism or spectrum from the ADOS except 1 who was subsequently removed from the analyses. Seven participants were excluded from the analyses because of excessive movement artifact (see below for description) resulting in a final sample of 14 participants with ASD (Table 1). Information about co-morbid diagnoses and current medications were obtained through a phone screen with either the participant or parent if the participant was a minor. This information was not available for 2 of the 14 ASD participants. Six of the 12 participants reported use of medications associated with symptoms of neuropsychiatric disorders [ADHD (4), depression/anxiety (3), psychosis (2)]. Only two participants, however, reported any co-morbid neurological disorders and these were obsessive-compulsive disorder (1) and attention deficit hyperactivity disorder (2).

\section{NEUROTYPICAL PARTICIPANTS}

Twenty-three NT participants (14-20 years; all male) performed a resting-state scan. Participants were excluded if they reported any psychiatric or neurological disorders on a selfreport screening questionnaire, which was filled out either by the participant or the parent. To screen for the presence of autism or autistic-like traits in the typical population, the participant's parents completed the SCQ screening described above. One participant who was no longer a minor completed the Autism Spectrum Quotient (AQ; Woodbury-Smith et al., 2005). No included participants received scores above the suggested threshold for autism screening. One was excluded due to excessive movement. Of the 22 remaining participants, 14 were matched as closely as possible to the ASD group on age. IQ scores did not differ significantly between groups (see Table 1).

\section{MRI DATA ACOUISITION}

Participants came to the Athinoula A. Martinos Imaging Center at the McGovern Institute for Brain Research at MIT for MRI data collection on a 3T Siemens Magnetom Tim Trio Scanner. We collected a structural MPRAGE image (128 sagittal slices, $\mathrm{TE}=3.39 \mathrm{~ms}, \mathrm{TR}=25 \mathrm{~ms}$, voxel size $1.3 \mathrm{~mm} \times 1 \mathrm{~mm} \times 1.3 \mathrm{~mm})$ and a resting-state functional MRI scan (67 sagittal slices, $\mathrm{TE}=30 \mathrm{~ms}, \mathrm{TR}=6000 \mathrm{~ms}$, $\#$ of TRs $=64$, voxel size $=2.0 \mathrm{~mm}$ isotropic) as part of a 90-min battery of tasks examining social processing that are not presented here. The last scan of the battery was the resting-state scan for which we asked participants to remain still with eyes open and fixated on a cross in the center of the screen. We chose a $6 \mathrm{~s}$ TR for the resting-state scan in order to achieve high spatial resolution with whole-brain coverage because previous work has demonstrated that array coils provide the biggest increases in temporal signal to noise ratio (tSNR) at high spatial resolutions (Triantafyllou et al., 2011). While this TR is unusually long, a study by Van Dijk et al. (2010), showed that there was no significant difference in the correlation strengths between the resting-state networks when compared between a TR of 2.5 and $5 \mathrm{~s}$.

\section{FUNCTIONAL MRI PREPROCESSING}

All data were analyzed using SPM8 ${ }^{1}$, Nipype (Gorgolewski et al., 2011), the CONN functional connectivity toolbox ver $13 \mathrm{e}^{2}$ (Whitfield-Gabrieli and Nieto-Castanon, 2012), and in-house Matlab (The Mathworks, Natick, MA, USA) scripts. All restingstate volumes were corrected for differences in the timing of slice acquisition. Functional data were realigned to the mean of all functional volumes in the timeseries using a $6^{\circ}$ rigid spatial transformation, which provided the spatial deviation for each timepoint for translational $(\mathrm{x}, \mathrm{y}, \mathrm{z})$ and rotational (roll, pitch, yaw) directions of movement. Functional data were then smoothed with a Gaussian smoothing kernel of $6 \mathrm{~mm}$ full-width half maximum, and normalized into standard Montreal Neurological Institute (MNI) space using non-linear transformations.

\footnotetext{
${ }^{1}$ www.fil.ion.ucl.ac.uk/spm

${ }^{2}$ http://www.nitrc.org/projects/conn/
} 


\section{ANALYSES OF HEAD MOTION}

The artifact detection toolbox $(\mathrm{ART})^{3}$ was used to examine outliers in global signal and movement for each participant. Timepoints were marked as outliers if global signal exceeded three standard deviations of the mean or if movement exceeded $1 \mathrm{~mm}$ (across translational and rotational directions) of scan-to-scan deviation. Participants for whom greater than $20 \%$ of the run was marked as an outlier were removed from the analyses (seven ASD; one NT). Head motion has been shown to result in spurious patterns of correlations (both increased and decreased; e.g., Power et al., 2011). Thus to examine whether groups differed as a function of head motion we used between-group $t$-tests to test for differences in (1) the total number of outliers and (2) the sum across all volumes of the absolute value of the deviation (in $\mathrm{mm}$ ) from the reference volume (i.e., the realignment parameters) for each of the six possible motion directions (i.e., $\mathrm{x}, \mathrm{y}, \mathrm{z}$, roll, pitch, yaw). Using between group $t$-tests, we also examined whether those participants who were excluded from the analyses due to excessive head motion were systematically different from those included in terms of age, IQ, or autism severity (Table 1). No significant differences in head motion between groups were present for either the number of outliers (see Table 1) or realignment parameters in any of the six directions [x: $t(24)=-0.56$, $p<0.58 ; \mathrm{y}: t(24)=-0.58, p<0.57 ; \mathrm{z}: t(24)=1.1, p<0.28$; roll: $t(24)=0.85, p<0.41$; pitch: $t(24)=0.18, p<0.86$, yaw: $t(24)=1.7, p<0.11)$. However, the ASD participants who were excluded due to excessive head motion had significantly lower Verbal Composite IQ scores, and higher (worse) social impairments as measured by the ADOS Reciprocal Social Interaction subscale and autism severity as measured by the Combined ADOS Communication and Reciprocal Social Interaction subscales. Excluded participants also showed a trend toward significantly younger ages (Table 1).

\section{FUNCTIONAL CONNECTIVITY ANALYSES}

To minimize the effects of head motion, whole-brain voxel-wise regression analyses were run for each seed region of interest with the six motion parameters from realignment and their temporal derivatives and each outlier timepoint entered separately as noise covariates. Additionally, using the aCompCor method (Behzadi et al., 2007) to account for physiological noise, covariates were included with a principal components analysis (PCA)-reduction (three dimensions) of the signal from white matter and CSF voxels based on each individual's unique segmented white matter and CSF masks. The residual datasets were then temporally filtered $(0.01<f<0.08)$ to focus analyses to the low-frequency oscillations characteristic of resting-state networks.

Whole-brain regression analyses were computed for each of the 34 seed regions of interest (Fair et al., 2009; Table 2) on the preprocessed, "clean" datasets for each participant. These analyses resulted in a correlation value in each voxel for each of the 34 seed regions. Normalized correlation values were created by a Fishers r-to-z transform and used in subsequent analyses. Averaging the normalized correlation coefficients within each group for each region pair created correlation matrices

\footnotetext{
${ }^{3}$ http://www.nitrc.org/projects/artifact_detect/
}

for each of the 34 regions of interest (ROI). Two-way between group (ASD vs. NT) $t$-tests were run for each of the 561 ROI-ROI pairs to examine whether differences in connectivity strength between groups were present and specific to particular networks. False discovery rate (FDR; $q<0.05$ ) was used to correct for multiple comparisons for the ROI-ROI comparisons.

Graph theory analyses were computed using the CONN functional connectivity toolbox. The unweighted ROI-to-ROI correlation matrices were first thresholded at a cost value of $k=0.15$. Cost is a measure of the proportion of connections for each ROI in relation to all connections in the network. Rather than determining a fixed correlation value as a threshold (e.g., $r=0.1$ ), using a cost threshold allows for roughly the same number of connections across participants by varying the correlation threshold for each participant to achieve the fixed cost threshold. When cost is equated across participants, direct comparisons across groups of network property differences can be made. Small world properties are observed in the range of costs $0.05<k<0.34$, where global efficiency is greater than that of a lattice graph and local efficiency is greater than that of a random graph (Achard and Bullmore, 2007). A cost threshold of .15 has also been demonstrated to provide a high degree of reliability when comparing session-specific estimates of graph theoretical measures across repeated runs or sessions (e.g., global efficiency $r=0.95$, local efficiency $r=0.9$; Whitfield-Gabrieli and Nieto-Castanon, 2012). We employed both one- and two-sided cost thresholds. In a one-sided cost threshold only positive correlations are considered, whereas two-sided includes both positive and negative correlations. To confirm that our findings generalize beyond these specific parameters, data were examined at a cost threshold of $0.05,0.1,0.2$, and 0.25 and compared to the findings with our a priori cost threshold of 0.15 .

The specific measures of interest were those of integration (global efficiency), segregation (local efficiency), and centrality (betweenness centrality). Between-group $t$-tests were used to compare network measures between groups with a FDR correction of $q<0.05$. Global efficiency is calculated as the average of the inverse of the shortest path length between each ROI (or node) and all other ROIs. The shortest path length is defined as the fewest number of connections (or correlations) between two nodes. Thus, a network with high global efficiency would be one in which nodes are highly integrated so the path between nodes is consistently short. With cost kept constant, this measure can be thought of as reflecting global, long-distance connections within the brain. Local efficiency is calculated as the average inverse of the shortest path length between the neighbors of any given node (or ROI). In other words, local efficiency measures the extent to which nodes are part of a cluster of locally, interconnected nodes. Finally, we examined a measure of centrality, betweenness centrality, which measures the fraction of all shortest path lengths in a network that pass through a given node. Thus, if a node is directly connected to many other nodes in the network it will have a shorter overall path length and function as a hub within and between networks. For more details on graph theoretical measures see Bullmore and Bassett (2011) or Rubinov and Sporns (2010). 
Table 2 | Seed regions of interest.

\begin{tabular}{|c|c|c|c|c|c|c|}
\hline Number & Network & Hemi & Region & $\mathbf{x}$ & $\mathbf{v}$ & $\mathbf{z}$ \\
\hline 1 & Cingulo-opercular & $\mathrm{L}$ & Anterior prefrontal cortex anterior insula/frontal & -28 & 51 & 15 \\
\hline 3 & Cingulo-opercular & L & Anterior thalamus dorsal anterior cingulate/medial superior & -12 & -15 & 7 \\
\hline 4 & Cingulo-opercular & $\mathrm{L}$ & Frontal cortex & -1 & 10 & 46 \\
\hline 7 & Cingulo-opercular & $\mathrm{R}$ & Anterior thalamus & 10 & -15 & 8 \\
\hline 8 & Cerebellar & L & Inferior cerebellum & -19 & -78 & -33 \\
\hline 9 & Cerebellar & $\mathrm{L}$ & Lateral cerebellum & -32 & -66 & -29 \\
\hline 10 & Cerebellar & $\mathrm{R}$ & Inferior cerebellum & 18 & -80 & -33 \\
\hline 14 & Default & L & Parahippocampal gyrus & -22 & -26 & -16 \\
\hline IS & Default & L & Superior fronta & -14 & 38 & 52 \\
\hline 16 & Default & $\mathrm{R}$ & Inferior temporal & 65 & -17 & -15 \\
\hline 17 & Default & $\mathrm{R}$ & Lateral parietal & 53 & -67 & 36 \\
\hline 18 & Default & $\mathrm{R}$ & Parahippocampal gyrus & 25 & -26 & -14 \\
\hline 19 & Default & $\mathrm{R}$ & Superior frontaanterior medial prefrontal & 17 & 37 & 52 \\
\hline 20 & Default & $\mathrm{R}$ & Cortex & 1 & 54 & 21 \\
\hline 21 & Default & L & Posterior cingulate cortex & -2 & -36 & 37 \\
\hline 22 & Default & $\mathrm{R}$ & Retrosplenial cortex ventromedial prefrontal & 3 & -51 & 8 \\
\hline 29 & Fronto-parietal & $\mathrm{R}$ & Inferior parietal lobe & 51 & -47 & 42 \\
\hline 30 & Fronto-parietal & $\mathrm{R}$ & Intraparietal sulcus & 30 & -61 & 39 \\
\hline 31 & Fronto-parietal & $\mathrm{R}$ & Dorsolateral prefrontal cortex & 43 & 22 & 34 \\
\hline 32 & Fronto-parietal & $\mathrm{R}$ & Frontal & 41 & 3 & 36 \\
\hline 33 & Fronto-parietal & $\mathrm{R}$ & Precuneus & 10 & -69 & 39 \\
\hline 34 & Fronto-parietal & LR & Mid cingulate cortex & 0 & -29 & 30 \\
\hline
\end{tabular}

These regions of interest and coordinates are taken directly from Fair etal. (2009). Number corresponds to the number listed in Figure 1.

\section{RESULTS}

\section{LARGELY TYPICAL NETWORK ORGANIZATION IN ASD}

Comparison of normalized correlation matrices between groups revealed minimal differences, which do not survive correction for multiple comparisons. Similarly network analyses revealed largely typical patterns of connectivity in the ASD group as compared to the NT group. Contrary to our hypotheses we found no differences in measures of global or local efficiency. Only betweenness centrality, which indicates the degree to which a seed (or node) functions as a hub within and between networks, was significantly different between groups and it was greater for participants with autism for the right lateral parietal (RLatP) seed of the DMN $(t(26)=3.52 ; p<0.027$ FDR-corrected) only. This metric was only significantly different when both positive and negative correlations were used in the cost threshold. When only positive correlations were considered, greater betweenness centrality in RLatP remained larger in ASD than NT groups but not significantly $(t(26)=1.57, p<0.13)$. This finding suggests both correlations and anti-correlations (i.e., negative correlations) drove differences between groups. This effect held when examining higher 
cost thresholds $(k=0.2$ and 0.25$)$ but not lower $(k=0.1$ and 0.05).

\section{EXPLORATION OF RIGHT LATERAL PARIETAL SEED CONNECTIVITY PATTERNS}

Comparison of the $34 \times 34$ matrix of normalized correlation values between seed regions for each group suggests the higher betweenness centrality in ASD may be due to (1) greater longdistance connectivity within the default mode network [RLatPanterior medial prefrontal cortex (aMPFC)] and (2) greater negative correlations with regions in cerebellar and control networks in participants with ASD (Figure 1). However, these ROI-toROI differences were not significant when controlling for multiple comparisons. To further investigate how differences in connectivity resulted in the difference in centrality between groups we conducted within- and between-group $t$-tests on correlation maps using the RLatP region as a seed region (Figure 2, Table 3). These maps demonstrate significantly greater functional connectivity in the ASD than NT group within medial prefrontal cortex using a FWE cluster correction of $p<0.05$. The NT group showed higher connectivity between the RLatP seed and cerebellar tonsils [a region previously associated with the default mode network (Fox et al., 2005)]. Examination of correlation maps within each group suggests these regions of between-group differences are not driven only by negative correlations in one group.

Our findings of greater connectivity within long-distance regions of the default mode network and greater centrality in autism were surprising and thus we explored whether variance in RLatP connectivity was related to autism severity, as measured by the ADOS, IQ, or age. No significant relationships were seen for autism severity or IQ and betweenness centrality measures for the RLatP, although there was a trend toward reduced centrality with

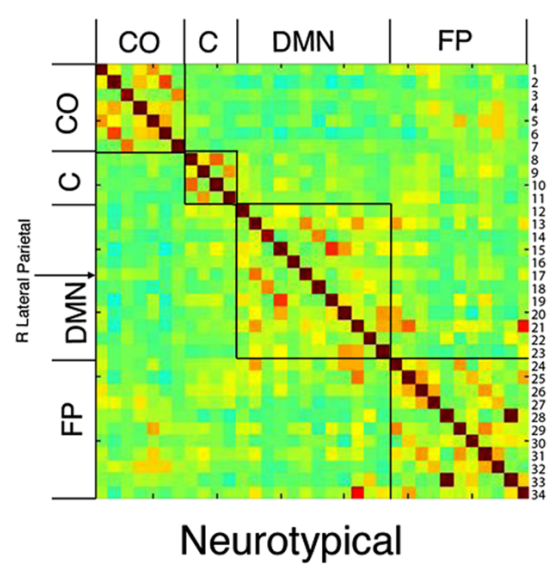

FIGURE 1 | Correlation matrices for neurotypical (A) and ASD (B) groups Normalized correlation coefficients are reported for each of the $34 \times 34 \mathrm{ROI}$ correlations by group. These are organized by network based on Fair et al. (2009) (CO, cingulo-opercular; C, cerebellar; DMN, default mode network; FP, fronto-parietal). Each row is labeled with a number which corresponds to 1 of

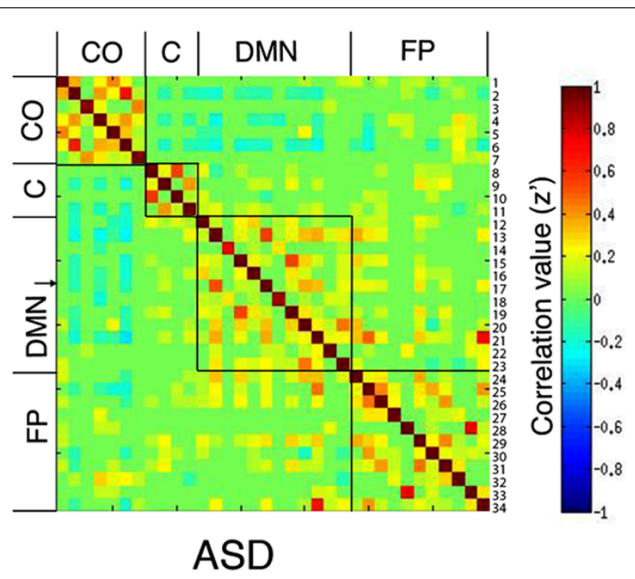

34 seed regions (see Table 2 for a list by number). Comparison of these matrices resulted in no significant differences between groups, when corrected for multiple comparisons. The right lateral parietal seed region (\#17) of the DMN is identified with an arrow because that region showed a significant effect of group on centrality measures.

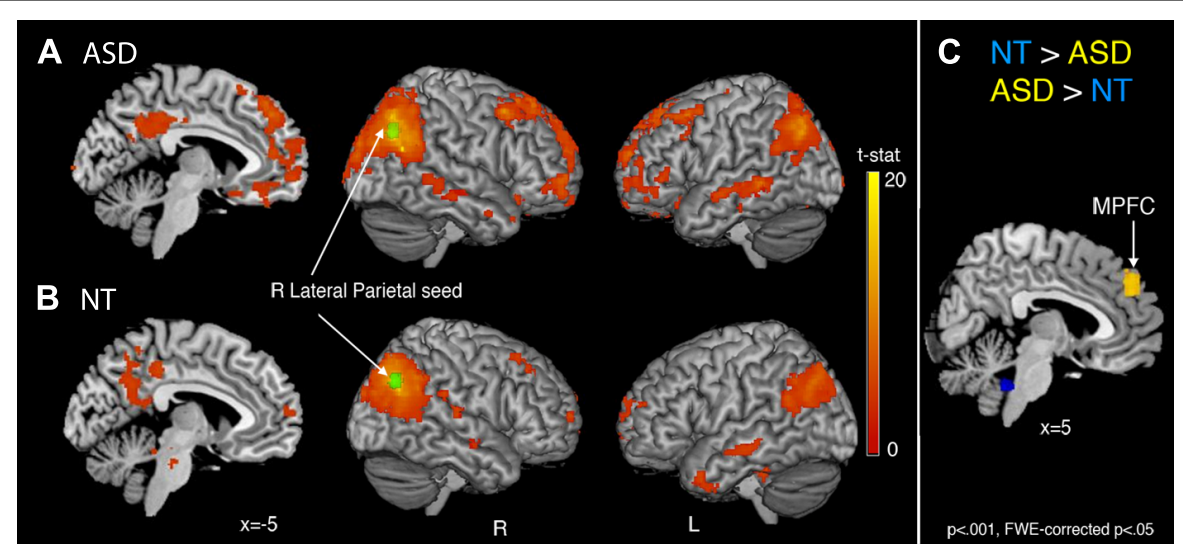

FIGURE 2 |Whole-brain functional connectivity maps with the right lateral parietal (RLatP) region (green) as a seed region are shown for the ASD group (A) and neurotypical group (B). Between-group comparisons (C) revealed one region of significantly greater connectivity from the RLatP seed in the ASD than NT group (yellow) which was the medial prefrontal cortex. The NT group showed greater connectivity between the RLatP seed and regions within the cerebellum (blue) than the ASD group. All maps are thresholded at $p<0.001$, FWE cluster corrected at $p<0.05$. 
Table 3 | Functional connectivity from the right lateral parietal seed region.

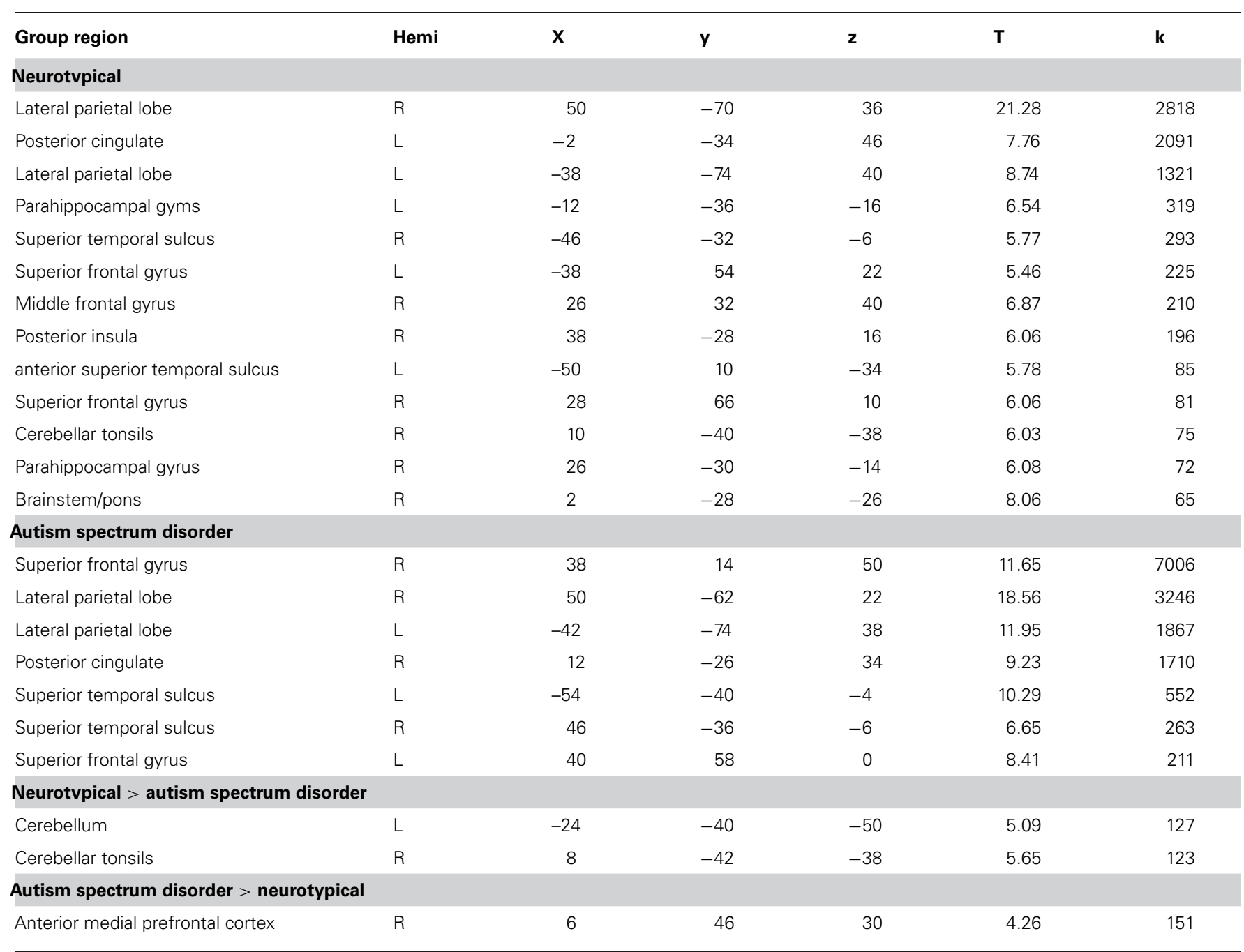

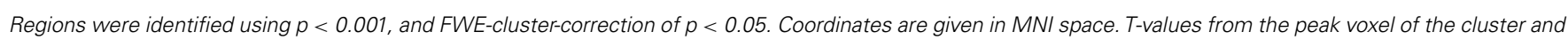
size $(k)$ of the cluster are given. Clusters are organized by size.

age in the ASD group only $[r(13)=-0.48, p<0.086)$. Because the aMPFC was a region that showed significantly increased connectivity with RLatP in ASD in whole-brain analyses, we examined whether the strength of connectivity between the RLatP seed and the aMPFC seed was correlated with ADOS scores, IQ, or age. We found a negative correlation between the ADOS combined social-communication subscale and RLatP to aMPFC connectivity $[r(13)=-0.56, p<0.046)$, which was driven by the communication subscale $[r(13)=-0.67, p<0.012)$, suggesting lower connectivity within long-distance regions of the default mode network is related to more severe autism (Figure 3). No other correlations reached significance.

\section{DISCUSSION}

Overall, these data are consistent with recent studies suggesting largely typical patterns of functional connectivity in individuals with autism (Tyszka et al., 2013). Although network organization across four functional networks was examined, this relatively high-functioning group of adolescent males demonstrated only one significant difference in graph theoretical metrics of network organization: namely, betweenness centrality of the RLatP region of the DMN. Follow-up whole-brain voxel-wise analyses with the RLatP region as a seed region revealed greater connectivity in ASD to another region of the DMN, the aMPFC, as compared to NT controls.

Of the four functional networks examined in the current study, the DMN is the most consistently implicated in autism - though that may be largely due to a bias in the number of studies investigating this network alone. The DMN comprises a set of regions showing deactivation during goal-directed tasks, higher metabolic activity during rest, and relative activation during tasks requiring internally directed thought or social processing (e.g., Gusnard and Raichle, 2001). In autism, however, these regions do not show the typical pattern of deactivation during goal-directed tasks (Kennedy etal., 2006; Murdaugh etal., 2012) and show reduced activation during tasks of social-cognitive processing (e.g., 


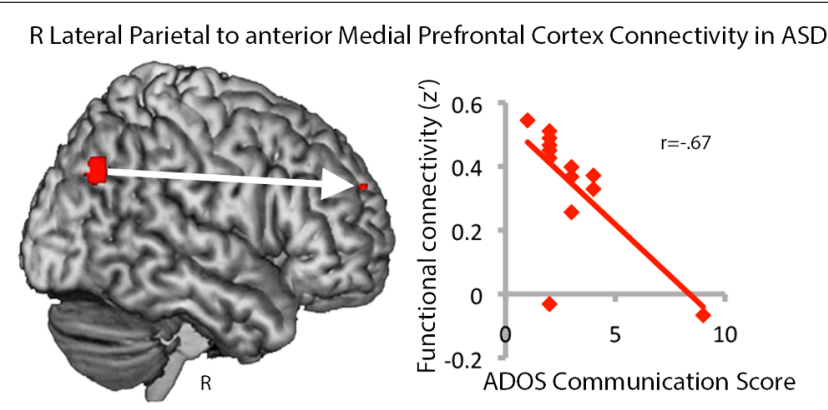

FIGURE 3 | Functional connectivity between the right lateral parietal and anterior medial prefrontal cortex regions in the default mode network is negatively correlated with ADOS communication scores in the ASD group. Higher scores indicate greater impairment.

Gilbert et al., 2009; Murdaugh et al., 2012, but see Dufour et al., in press). Furthermore, many previous studies have found a pattern of reduced DMN functional connectivity in ASD, particularly between long-distance frontal and parietal regions (Kennedy and Courchesne, 2008; Monk et al., 2009; Assaf et al., 2010; Weng et al., 2011; Murdaugh et al., 2012; Rudie et al., 2012; von dem Hagen et al., 2013, but see Lynch et al., 2013). Thus, while findings of atypical engagement of the DMN in autism is not new, the finding of greater functional connectivity between RLatP and medial prefrontal regions of the default mode network in ASD is inconsistent with many previous studies.

There are (at least) two factors that may account for differences between our study and previous studies finding reduced connectivity between groups. First, we matched groups on head motion parameters and used two measures to account for uncorrected head motion in subsequent analyses. While some previous studies demonstrated no significant differences in head motion between groups, four of the seven studies that showed reduced functional connectivity in the DMN did not compare head motion across groups. Differences in head motion between groups is a critical factor as previous studies have suggested that head motion may account for systematic and spurious correlations, particularly in reducing long-distance correlations while increasing short-distance correlations (Power etal., 2011). It remains unclear if "accounting" for head motion in the analysis is sufficient to eliminate group differences that may be due to motion.

Second, our final sample consisted of quite high-functioning individuals with autism. Many previous studies reporting reduced functional connectivity had, on average, slightly higher ADOS scores and lower IQs. Further, within the current study a significant relationship was found between functional connectivity between RLatP and MPFC and ADOS combined social-communication (and communication) scores, with greater impairment relating to lower functional connectivity. Taken together, these findings suggest lower-functioning autism may result in patterns of reduced connectivity. However, we offer caution in this interpretation because this relationship is counterintuitive in the context of the current study. The ASD group had significantly greater connectivity than the NT group, which suggests that more severe autism should be related to greater connectivity, but instead the reverse is true. These data suggest a possible non-linear relationship between autism severity and functional connectivity in autism but this has yet to be systematically examined.

Systematically examining how level of functioning impacts connectivity patterns is especially challenging because lowerfunctioning individuals tend to have more motion artifact, and, as discussed above, head motion differences alone can lead to a pattern of reduced long-distance connectivity. In the current study, we used stringent criteria to exclude participants with excessive head motion and while this only resulted in loss of data from one NT participant, seven participants with ASD were removed from data analyses. These seven were significantly different from the rest of the ASD group not only because they moved more during the scan but also because they were younger, had higher ADOS scores (i.e., were more impaired), and had lower verbal and composite IQ scores. Thus, a significant, but necessary, challenge for further research is to characterize the functional significance of restingstate networks when head motion is equated across groups (Deen and Pelphrey, 2012), such as in the current study.

Although less common, this is not the first study to report hyper-connectivity within the default mode network in autism. Two previous studies also reported increased connectivity in ASD within default mode regions (Monk et al., 2009; Lynch et al., 2013), and for one (Lynch et al., 2013) this increased connectivity was found between frontal and parietal DMN regions similar to the current study. Specifically, Lynch et al. (2013) examined functional connectivity from regions within posteromedial cortex in 7-12-year-old children and reported greater connectivity in ASD from retrosplenial cortex, a region just inferior to the posterior cingulate and part of the default mode network, to several other regions including the aMPFC (though this particular connection was reduced in the ASD sample in Monk et al., 2009). Additionally, connectivity between posterior cingulate and several lateral and medial temporal regions showed greater connectivity in the ASD than NT groups - a finding similar to Monk et al. (2009).

The study of Lynch et al. (2013) was among the first to examine DMN connectivity during a resting baseline in young children with ASD. As such, they suggested the relatively novel finding of hyper-connectivity within the default mode network (and from posteromedial cortex to regions outside of the DMN) may be due to a developmental change in the pattern of connectivity differences between ASD and NT groups. This developmental story is consistent with other theories of connectivity in autism (e.g., Courchesne and Pierce, 2005; Pelphrey et al., 2011) as well as evidence of age-related changes in brain differences between autism and control groups (Redcay and Courchesne, 2005). In other words, whereas findings from older children and adults reveal reduced brain size, reduced measures of white matter integrity (e.g., FA) or reduced functional connectivity, findings from younger children reveal larger brain size (e.g., Courchesne et al., 2001; Hazlett et al., 2006), higher FA values (Wolff et al., 2012), and increased functional connectivity (Lynch et al., 2013). However, the current findings of DMN hyper-connectivity was in a sample of adolescents and the Monk et al. (2009) study was in 
adults. Thus, age-related differences may not completely account for patterns of increased functional connectivity within the default mode network.

While further research is needed to disentangle the factors contributing to relatively typical or increased connectivity in autism, we find the increased connectivity between the RLatP and aMPFC regions of the DMN in the current study intriguing. These regions play an important role in social processes that are atypical in individuals with autism, including mental state judgments of others (i.e., theory of mind) and of one's self (i.e., introspection) (e.g., Baron-Cohen et al., 1985; Frith and Happe, 1999; Saxe and Kanwisher, 2003; Saxe et al., 2006; Senju et al., 2009). While the medial prefrontal cortex plays a general role in mentalizing (WhitfieldGabrieli et al., 2011), portions of RLatP cortex may play a more specific role in thinking about others thoughts and beliefs, or theory of mind (e.g., Saxe and Kanwisher, 2003; Saxe et al., 2006). Meta-analyses suggest the RLatP region of the default mode is at least partially overlapping with the right temporoparietal junction (RTPJ) often reported in studies of theory of mind processing (e.g., Schilbach et al., 2008; Spreng and Mar, 2012). Beyond socialcognitive processing, the RLatP lobe is also associated with shifts of spatial attention (Corbetta and Shulman, 2002), semantic processing (Binder et al., 1999), and narrative comprehension (e.g., Mar, 2011), all of which have been implicated as atypical in individuals with autism. Thus, greater connectivity within right parietal cortex could indicate less functional specialization of this region in ASD, similar to findings of right posterior temporal cortex (e.g., Shih et al., 2011). However, the current data do not directly address that hypothesis.

A notable limitation in this study, which claims minimal differences in functional connectivity between groups, is a small sample size. Nonetheless, the current findings of greater connectivity

\section{REFERENCES}

Achard, S., and Bullmore, E. (2007). Efficiency and cost of economical brain functional networks. PLoS Comput. Biol. 3:e17. doi: 10.1371/journal.pcbi.0030017

Anderson, J. S., Druzgal, T. J., Froehlich, A., DuBray, M. B., Lange, N., Alexander, A. L., et al. (2011). Decreased interhemispheric functional connectivity in autism. Cereb. Cortex 21, 1134-1146. doi: 10.1093/cercor/bhq190

Assaf, M., Jagannathan, K., and Calhoun, V. (2010). Abnormal functional connectivity of default mode sub-networks in autism spectrum disorder patients. Neuroimage 53, 247-256. doi: 10.1016/ j.neuroimage.2010.05.067.Assaf

Barnea-Goraly, N., Kwon, H., Menon, V., Eliez, S., Lotspeich, L., and Reiss, A. L. (2004). White matter structure in autism: preliminary evidence from diffusion tensor imaging. Biol. Psychiatry 55, 323-326. doi: 10.1016/j.biopsych.2003.10.022

Baron-Cohen, S., Leslie, A. M., and Frith, U. (1985). Does the autistic

within the DMN in ASD adds to the small, growing body of literature suggesting inconsistent support for an underconnectivity theory of autism. A second limitation is the restricted range of high-functioning participants with autism who were able to complete the scan with minimal motion artifact. Even within this narrow range, a correlation was seen between a greater level of communicative impairment and lower functional connectivity between RLatP and medial prefrontal cortex and a trend toward increasing age and reduced betweenness centrality in ASD. Finally, a third limitation is the inclusion of data from participants currently on medication as some medications may affect the strength or patterns or brain activation; however, the sample is too small to determine whether medication had any systematic effects on functional connectivity. These data underscore the need for developmental studies of functional connectivity in high- and low-functioning individuals with autism in which head motion is tightly matched between groups.

\section{ACKNOWLEDGMENTS}

We gratefully acknowledge the Boston Autism Consortium for providing funding support for this project. We also wish to thank Dr. Charles A. Nelson, Dr. Tal Kenet, and Dr. Robert Joseph for their contributions to this multi-site project that made collection of these data possible, including recruitment and assessment of participants with ASD. We also thank Dr. Jasmin Cloutier and Daniel O'Young for assistance with data collection and the Athinoula A. Martinos Imaging Center at the McGovern Institute for Brain Research at MIT, particularly Dr. Christina Triantafyllou for development of the current resting-state imaging protocol. We also are grateful to the Eunice Kennedy Shriver National Institute of Child Health and Human Development for a postdoctoral fellowship to Elizabeth Redcay.

J. Cogn. Neurosci. 11, 80-95. doi: 10.1162/089892999563265

Biswal, B., Yetkin, F. Z., Haughton, V. M., and Hyde, J. S. (1995). Functional connectivity in the motor cortex of resting human brain using echo-planar MRI. Magn. Reson. Med. 34, 537-541. doi: 10.1002/mrm. 1910340409

Bullmore, E. T., and Bassett, D. S. (2011). Brain graphs: graphical models of the human brain connectome. Annu. Rev. Clin. Psychol. 7, 113140. doi: 10.1146/annurev-clinpsy040510-143934

Cherkassky, V. L., Kana, R. K., Keller, T. A., and Just, M. A. (2006). Functional connectivity in a baseline restingstate network in autism. Neuroreport 17, 1687-1690. doi: 10.1097/ 01.wnr.0000239956.45448.4c

Corbetta, M., and Shulman, G. L. (2002). Control of goal-directed and stimulus-driven attention in the brain. Nat. Rev. Neurosci. 3, 201-215. doi: 10.1038/nrn755

Corsello, C., Hus, V., Pickles, A., Risi, S., Cook, E. H., Leventhal, B. L., etal. (2007). Between a ROC and a hard place: decision making and making decisions about using the SCQ. J. Child Psychol. Psychiatry 48, 932-940. doi: 10.1111/j.14697610.2007.01762.x

Courchesne, E., Karns, C. M., Davis, H. R., Ziccardi, R., Carper, R. A., Tigue, Z. D., et al. (2001). Unusual brain growth patterns in early life in patients with autistic disorder: an MRI study. Neurology 57, 245-254. doi: 10.1212/WNL.57.2.245

Courchesne, E., and Pierce, K. (2005). Why the frontal cortex in autism might be talking only to itself: local over-connectivity but long-distance disconnection. Curr. Opin. Neurobiol. 15, 225-230. doi: 10.1016/j.conb.2005.03.001

Courchesne, E., Pierce, K., Schumann, C. M., Redcay, E., Buckwalter, J. A., Kennedy, D. P., et al. (2007). Mapping early brain development in autism. Neuron 56, 399-413. doi: 10.1016/j.neuron.2007.10.016

Damoiseaux, J. S., Rombouts, S. A. R. B., Barkhof, F., Scheltens, P., Stam, C. J., Smith, S. M., et al. (2006). Consistent resting-state networks across 
healthy subjects. Proc. Natl. Acad. Sci. U.S.A. 103, 13848-13853. doi: $10.1073 /$ pnas.0601417103

Deen, B., and Pelphrey, K. (2012). Perspective: brain scans need a rethink. Nature 491, S20. doi: 10.1038/ 491S20a

Di Martino, A., Kelly, C., Grzadzinski, R., Zuo, X.-N., Mennes, M., Mairena, M. A., et al. (2011). Aberrant striatal functional connectivity in children with autism. Biol. Psychiatry 69, 847-856. doi: 10.1016/ j.biopsych.2010.10.029.Aberrant

Di Martino, A., Ross, K., Uddin, L. Q., Sklar, A. B., Castellanos, F. X., and Milham, M. P. (2009). Functional brain correlates of social and nonsocial processes in autism spectrum disorders: an activation likelihood estimation meta-analysis. Biol. Psychiatry 65, 63-74. doi: 10.1016/j.biopsych.2008.09.022

Dinstein, I., Pierce, K., Eyler, L., Solso, S., Malach, R., Behrmann, M., etal. (2011). Disrupted neural synchronization in toddlers with autism. Neuron 70, 1218-1225. doi: 10.1016/j.neuron.2011.04.018

Dosenbach, N. U. F., Visscher, K. M., Palmer, E. D., Miezin, F. M., Wenger, K. K., Kang, H. C., et al. (2006). A core system for the implementation of task sets. Neuron 50, 799-812. doi: 10.1016/j.neuron.2006.04.031

Dufour, N., Redcay, E., Young, L., Mavros, P. L., Moran, J. M., Triantafyllou, C., et al. (in press). Similar brain activation patterns during false belief tasks in a large sample of adults with and without autism. PLoS ONE.

Ebisch, S. J. H., Gallese, V., Willems, R. M., Mantini, D., Groen, W. B., Romani, G. L., et al. (2011). Altered intrinsic functional connectivity of anterior and posterior insula regions in high-functioning participants with autism spectrum disorder. Hum. Brain Mapp. 32, 1013-1028. doi: 10.1002/hbm.21085

Emerson, R. W., and Cantlon, J. F, (2012). Early math achievement and functional connectivity in the frontoparietal network. Dev. Cogn. Neurosci. 2(Suppl. 1), S139-S151. doi: 10.1016/j.dcn.2011.11.003

Fair, D. A., Cohen, A. L., Dosenbach, N. U. F., Church, J. A., Miezin, F. M., Barch, D. M., et al. (2008). The maturing architecture of the brain's default network. Proc. Natl. Acad. Sci. U.S.A. 105, 4028-4032. doi: 10.1073/pnas.0800376105

Fair, D. A., Cohen, A. L., Power, J. D., Dosenbach, N. U. F., Church, J. A., Miezin, F. M., etal. (2009). Functional brain networks develop from a "local to distributed" organization.
PLoS Comput. Biol. 5:e1000381. doi: 10.1371/journal.pcbi.1000381

Fox, M. D., Snyder, A. Z., Vincent, J. L., Corbetta, M., Van Essen, D. C., and Raichle, M. E. (2005). The human brain is intrinsically organized into dynamic, anticorrelated functional networks. Proc. Natl. Acad. Sci. U.S.A. 102, 9673-9678. doi: 10.1073/pnas.0504136102

Frith, U., and Happe, F. (1999). Theory of mind and self-consciousness: what is it like to be autistic? Mind Lang. 14, 82-89. doi: 10.1111/14680017.00100

Gilbert, S. J., Meuwese, J. D. I., Towgood, K. J., Frith, C. D., and Burgess, P. W. (2009). Abnormal functional specialization within medial prefrontal cortex in high-functioning autism: a multi-voxel similarity analysis. Brain 132, 869-878. doi: 10.1093/brain/awn365

Gorgolewski, K., Burns, C. D., Madison, C., Clark, D., Halchenko, Y. O., Waskom, M. L., et al. (2011). Nipype: a flexible, lightweight and extensible neuroimaging data processing framework in python. Front. Neuroinform. 5:13. doi: 10.3389/fninf.2011. 00013

Gusnard, D. A., and Raichle, M. E. (2001). Searching for a baseline: functional imaging and the resting human brain. Nat. Rev. Neurosci. 2 , 685-694. doi: 10.1038/35094500

Hazlett, H. C., Poe, M. D., Gerig, G., Smith, R. G., and Piven, J. (2006). Cortical gray and white brain tissue volume in adolescents and adults with autism. Biol. Psychiatry 59, 1-6. doi: 10.1016/j.biopsych.2005. 06.015

Herbert, M. R., Ziegler, D. A., Makris, N., Filipek, P. A., Kemper, T. L., Normandin, J. J., et al. (2004). Localization of white matter volume increase in autism and developmental language disorder. Ann. Neurol. 55, 530-540. doi: 10.1002/ ana.20032

Just, M. A., Cherkassky, V. L., Keller, T. A., Kana, R. K., and Minshew, N. J. (2007). Functional and anatomical cortical underconnectivity in autism: evidence from an FMRI study of an executive function task and corpus callosum morphometry. Cereb. Cortex 17, 951-961. doi: 10.1093/cercor/bhl006

Just, M. A., Cherkassky, V. L., Keller, T. A., and Minshew, N. J. (2004). Cortical activation and synchronization during sentence comprehension in high-functioning autism: evidence of underconnectivity. Brain 127(Pt 8), 1811-1821. doi: 10.1093/brain/awh199
Kana, R. K., Keller, T. A., Cherkassky, V. L., Minshew, N. J., and Just, M. A. (2006). Sentence comprehension in autism: thinking in pictures with decreased functional connectivity. Brain 129(Pt 9), 2484-2493. doi: 10.1093/brain/awl164

Kana, R., Libero, L., Hu, C., Deshpande, H., and Colburn, J. (2012). Functional brain networks and white matter underlying theory-of-mind in autism. Soc. Cogn. Affect. Neurosci. doi: 10.1093/scan/nss106 [Epub ahead of print].

Kennedy, D. P., and Courchesne, E. (2008). The intrinsic functional organization of the brain is altered in autism. Neuroimage 39, 1877-1885. doi: 10.1016/j.neuroimage.2007.10.052

Kennedy, D. P., Redcay, E., and Courchesne, E. (2006). Failing to deactivate: resting functional abnormalities in autism. Proc. Natl. Acad. Sci. U.S.A. 103, 8275-8280. doi: 10.1073/pnas.0600674103

Khan, S., Gramfort, A., Shetty, N. R., Kitzbichler, M. G., Ganesan, S. Moran, J. M., et al. (2013). Local and long-range functional connectivity is reduced in concert in autism spectrum disorders. Proc. Natl. Acad. Sci. U.S.A. 110, 3107-3112. doi: 10.1073/pnas.1214533110

Lee, J. E., Bigler, E. D., Alexander, A. L., Lazar, M., DuBray, M. B., Chung, M. K., et al. (2007). Diffusion tensor imaging of white matter in the superior temporal gyrus and temporal stem in autism. Neurosci. Lett. 424 127-132. doi: 10.1016/j.neulet.2007. 07.042

Lewis, C. M., Baldassarre, A., Committeri, G., Romani, G. L., and Corbetta, M. (2009). Learning sculpts the spontaneous activity of the resting human brain. Proc. Natl. Acad. Sci. U.S.A. 106, 17558-17563. doi: 10.1073/pnas.0902455106

Lord, C., Risi, S., Lambrecht, L., Cook, E. H., Leventhal, B. L., DiLavore, P. C., et al. (2000). The autism diagnostic observation schedule-generic: a standard measure of social and communication deficits associated with the spectrum of autism. J. Autism Dev. Disord. 30, 205-223. doi: 10.1023/A:1005592 401947

Lynch, C. J., Uddin, L. Q., Supekar, K., Khouzam, A., Phillips, J., and Menon, V. (2013). Default mode network in childhood autism: posteromedial cortex heterogeneity and relationship with social deficits. Biol. Psychiatry 74, 212 219. doi: 10.1016/j.biopsych.2012. 12.013
Mar, R. A. (2011). The neural bases of social cognition and story comprehension. Annu. Rev. Psychol. 62, 103-134. doi: 10.1146/annurevpsych-120709-145406

Mason, R., Williams, D., Kana, R., and Minshew, N. (2008). Theory of mind disruption and recruitment of the right hemisphere during narrative comprehension in autism. Neuropsychologia 46, 269-280. doi: 10.1016/ j.neuropsychologia.2007.07.018

Minshew, N., and Keller, T. (2010). The nature of brain dysfunction in autism: functional brain imaging studies. Curr. Opin. Neurol. 23, 124-130. doi: 10.1097/WCO.0b013e32833782d4

Mizuno, A., Villalobos, M. E., Davies, M. M., Dahl, B. C., and Müller, R.-A. (2006). Partially enhanced thalamocortical functional connectivity in autism. Brain Res. 1104, 160-174. doi: 10.1016/j.brainres.2006.05.064

Monk, C., Peltier, S., and Wiggins, J. (2009). Abnormalities of intrinsic functional connectivity in autism spectrum disorders. Neuroimage 47, 764-772. doi: 10.1016/j.neuroimage.2009.04.069. Abnormalities

Müller, R.-A., Shih, P., Keehn, B., Deyoe, J. R., Leyden, K. M., and Shukla, D. K. (2011). Underconnected, but how? A survey of functional connectivity MRI studies in autism spectrum disorders. Cereb. Cortex 21, 2233-2243. doi: 10.1093/cercor/bhq296

Murdaugh, D. L., Shinkareva, S. V., Deshpande, H. R., Wang, J., Pennick, M. R., and Kana, R. K. (2012). Differential deactivation during mentalizing and classification of autism based on default mode network connectivity. PloS ONE 7:e50064. doi: 10.1371/journal.pone. 0050064

Murphy, E. R., Foss-Feig, J., Kenworthy, L., Gaillard, W., and Vaidya, C. J. (2012). Atypical functional connectivity of the amygdala in childhood autism spectrum disorders during spontaneous attention to eyegaze. Autism Research and Treatment. doi:10.1155/2012/652408

Nair, A., Treiber, J. M., Shukla, D. K., Shih, P., and Müller, R.-A. (2013). Impaired thalamocortical connectivity in autism spectrum disorder: a study of functional and anatomical connectivity. Brain 136(Pt 6), 19421955. doi: 10.1093/brain/awt079

Noonan, S., Haist, F., and Müller, R. (2009). Aberrant functional connectivity in autism: evidence from lowfrequency bold fluctuations. Brain Res. 1262, 48-63. doi: 10.1016/ j.brainres.2008.12.076.Aberrant 
Pelphrey, K. A., Shultz, S., Hudac, C. M., and Vander Wyk, B. C. (2011). Research review: constraining heterogeneity: the social brain and its development in autism spectrum disorder. J. Child Psychol. Psychiatry 52, 631-644. doi: 10.1111/j.14697610.2010.02349.x

Power, J. D., Barnes, K. A., Snyder, A. Z., Schlaggar, B. L., and Petersen, S. E. (2011). Spurious but systematic correlations in functional connectivity MRI networks arise from subject motion. Neuroimage 59, 2142-2154. doi: 10.1016/j.neuroimage.2011.10.018

Power, J. D., Fair, D. A., Schlaggar, B. L., and Petersen, S. E. (2010). The development of human functional brain networks. Neuron 67, 735-748. doi: 10.1016/j.neuron.2010.08.017

Redcay, E., and Courchesne, E. (2005). When is the brain enlarged in autism? A meta-analysis of all brain size reports. Biol. Psychiatry 58, 1-9. doi: 10.1016/j.biopsych.2005 . 03.026

Rippon, G., Brock, J., Brown, C., and Boucher, J. (2007). Disordered connectivity in the autistic brain: challenges for the "new psychophysiology". Int. J. Psychophysiol. 63, 164-172. doi: 10.1016/j. ijpsycho.2006.03.012

Rubinov, M., and Sporns, O. (2010). Complex network measures of brain connectivity: uses and interpretations. Neuroimage 52, 10591069. doi: 10.1016/j.neuroimage. 2009.10.003

Rudie, J. D., Hernandez, L. M., Brown, J. A., Beck-Pancer, D., Colich, N. L., Gorrindo, P., etal. (2012). Autism-associated promoter variant in MET impacts functional and structural brain networks. Neuron 75 , 904-915. doi: 10.1016/j.neuron.2012. 07.010

Saxe, R., and Kanwisher, N. (2003). People thinking about thinking people. The role of the temporoparietal junction in "theory of mind". Neuroimage 19, 1835-1842. doi: 10.1016/S1053-8119(03)00230-1

Saxe, R., Moran, J. M., Scholz, J., and Gabrieli, J. (2006). Overlapping and non-overlapping brain regions for theory of mind and self reflection in individual subjects. Soc. Cogn. Affect. Neurosci. 1, 229-234. doi: $10.1093 /$ scan/nsl034

Schilbach, L., Eickhoff, S. B., RotarskaJagiela, A., Fink, G. R., and Vogeley, K. (2008). Minds at rest? Social cognition as the default mode of cognizing and its putative relationship to the "default system" of the brain. Conscious. Cogn. 17, 457-467. doi: 10.1016/j.concog.2008.03.013

Senju, A., Southgate, V., White, S., and Frith, U. (2009). Mindblind eyes: an absence of spontaneous theory of mind in Asperger syndrome. Science $325,883-885$. doi: $10.1126 /$ science. 1176170

Shih, P., Keehn, B., Oram, J. K., Leyden, K. M., Keown, C. L., and Müller, R.-A. (2011). Functional differentiation of posterior superior temporal sulcus in autism: a functional connectivity magnetic resonance imaging study. Biol. Psychiatry. 70, 270-277. doi: 10.1016/j.biopsych. 2011.03.040

Spreng, R. N., and Mar, R. A. (2012). I remember you: a role for memory in social cognition and the functional neuroanatomy of their interaction. Brain Res. 1428, 43-50. doi: 10.1016/j.brainres.2010.12.024

Triantafyllou, C., Polimeni, J., and Wald, L. (2011). Physiological noise and signal-to-noise ratio in fmri with multi-channel array coils. Neuroimage 55, 597-606. doi: 10.1016/j.neuroimage.2010.11.084. Physiological

Tsiaras, V., Simos, P. G., Rezaie, R., Sheth, B. R., Garyfallidis, E., Castillo, E. M., et al. (2011). Extracting biomarkers of autism from MEG resting-state functional connectivity networks. Comput. Biol. Med. 41, 1166-1177. doi: 10.1016/j.compbiomed.2011.04.004

Turner, K. C., Frost, L., Linsenbardt, D., McIlroy, J. R., and Müller, R.-A. (2006). Atypically diffuse functional connectivity between caudate nuclei and cerebral cortex in autism. Behav. Brain Funct. 2, 34. doi: 10.1186/17449081-2-34

Tyszka, J. M., Kennedy, D. P., Paul, L. K., and Adolphs, R. (2013).
Largely typical patterns of restingstate functional connectivity in highfunctioning adults with autism. Cereb. Cortex doi: 10.1093/cercor/bht040

Van Dijk, K. R. A., Hedden, T., Venkataraman, A., Evans, K. C., Lazar, S. W., and Buckner, R. L. (2010). Intrinsic functional connectivity as a tool for human connectomics: theory, properties, and optimization. J. Neurophysiol. 103, 297-321. doi: 10.1152/jn.00783.2009 von dem Hagen, E. A. H., Stoyanova, R. S., Baron-Cohen, S., and Calder, A. J. (2013). Reduced functional connectivity within and between "social" resting state networks in autism spectrum conditions. Soc. Cogn. Affect. Neurosci. 8, 694-701. doi: 10.1093/scan/nss053

Wang, L., Laviolette, P., O'Keefe, K., Putcha, D., Bakkour, A., Van Dijk, K. R. A., et al. (2010). Intrinsic connectivity between the hippocampus and posteromedial cortex predicts memory performance in cognitively intact older individuals. Neuroimage 51, 910-917. doi: 10.1016/j.neuroimage. 2010.02.046

Washington, S. D., Gordon, E. M. Brar, J., Warburton, S., Sawyer, A T., Wolfe, A., etal. (2013). Dysmaturation of the default mode network in autism. Hum. Brain Mapp. doi:10.1002/hbm.22252 [Epub ahead of print].

Weng, S., Wiggins, J. L., Peltier, S. J., Carrasco, M., Risi, S., Lord, C., etal. (2011). Alterations of resting state functional connectivity in the default network in adolescents with autism spectrum disorders. Brain Res. 131, 202-214. doi: 10.1016/j.brainres.2009.11.057

Whitfield-Gabrieli, S., Moran, J. M., Nieto-Castañón, A., Triantafyllou, C., Saxe, R., and Gabrieli, J. D. E. (2011). Associations and dissociations between default and selfreference networks in the human brain. Neuroimage 55, 225-232. doi: 10.1016/j.neuroimage.2010.11.048

Whitfield-Gabrieli, S., and NietoCastanon, A. (2012). Conn: a functional connectivity toolbox for correlated and anticorrelated brain networks. Brain Connect. 2, 125-141. doi: 10.1089/brain.2012.0073

Wolff, J., Gu, H., and Gerig, G. (2012). Differences in white matter fiber tract development present from 6 to 24 months in infants with autism. Am. J. Psychiatry 169, 589-600. doi: 10.1176/appi.ajp.2011. 11091447

Woodbury-Smith, M. R., Robinson, J., Wheelwright, S., and BaronCohen, S. (2005). Screening adults for Asperger Syndrome using the AQ: a preliminary study of its diagnostic validity in clinical practice. $J$. Autism Dev. Disord. 35, 331-335. doi: 10.1007/s10803-005-3300-7

Zhu, Q., Zhang, J., Luo, Y. L. L., Dilks, D. D., and Liu, J. (2011). Resting-state neural activity across face-selective cortical regions is behaviorally relevant. J. Neurosci. 31, 10323-10330. doi: 10.1523/ JNEUROSCI.0873-11.2011

Conflict of Interest Statement: The authors declare that the research was conducted in the absence of any commercial or financial relationships that could be construed as a potential conflict of interest.

Received: 16 June 2013; accepted: 26 August 2013; published online: 19 September 2013.

Citation: Redcay E, Moran JM, Mavros PL, Tager-Flusberg H, Gabrieli JDE and Whitfield-Gabrieli S (2013) Intrinsic functional network organization in highfunctioning adolescents with autism spectrum disorder. Front. Hum. Neurosci. 7:573. doi: 10.3389/fnhum.2013.00573

This article was submitted to the journal Frontiers in Human Neuroscience. Copyright (c) 2013 Redcay, Moran, Mavros, Tager-Flusberg, Gabrieli and Whitfield-Gabrieli. This is an open-access article distributed under the terms of the Creative Commons Attribution License (CC BY). The use, distribution or reproduction in other forums is permitted, provided the original author(s) or licensor are credited and that the original publication in this journal is cited, in accordance with accepted academic practice. No use, distribution or reproduction is permitted which does not comply with these terms. 